\title{
Direct Digital Control of Single-Phase AC/DC PWM Converter System
}

\author{
Young Chol Kim ${ }^{\dagger}$, Lihua Jin*, Jinmok Lee**, and Jaeho Choi* \\ ${ }^{\dagger *}$ College of Electrical \& Computer Engineering, Chungbuk National University, Cheongju, Korea \\ ${ }^{* *}$ Automation R\&D Center, LS Industrial Systems, Geonggi, Korea
}

\begin{abstract}
This paper presents a new technique for directly designing a linear digital controller for a single-phase pulse width modulation (PWM) converter systems, based on closed-loop identification. The design procedure consists of three steps. First, obtain a digital current controller for the inner loop system by using the error space approach, so that the power factor of the supply is close to one. The outer loop is composed of a voltage controller, a current control loop including a current controller, a PWM converter, and a capacitor. Then, all the components, except the voltage controller, are identified by a discrete-time equivalent linear model, using the closed-loop output error (CLOE) method. Based on this equivalent model, a proper digital voltage controller is then directly designed. It is shown through PSim simulations and experimental results that the proposed method is useful for the practical design of PWM converter controllers.
\end{abstract}

Key Words: direct digital control, AC/DC PWM converter, closed-loop identification, characteristic ratio assignment (CRA)

\section{INTRODUCTION}

PWM AC/DC converters have been widely used for applications such as PWM inverter fed induction motor drives and interruptible power supply (UPS) systems. The main objectives of a PWM AC/DC converter are to regulate the DC voltage level while maintaining a sinusoidal AC current waveform and a unity power factor.

For the control of voltage source converters, the current controlled PWM techniques have been widely used due to their advantages, such as the control of instantaneous current waveforms and high accuracy, peak current protection, extremely good dynamics, and compensation of effects due to parameter changes and $\mathrm{AC}$ source voltage changes, etc. [1]. Current controlled PWM techniques can be divided into two groups: linear and nonlinear. The linear controllers include the ramp comparison current controller [2], the synchronous vector controller [3], the state feedback controller [4], and the predictive and dead-beat controllers [5], [6]. The hysteresis controller [2], the pulse density modulation algorithm [7], and the neural networks and fuzzy logic based controllers belong to the nonlinear controller group [8], [9]. Each controller has its advantages and limitations, but as the trend favors fully digital control, the methods which allow digital implementation are preferred, despite some sacrifices in accuracy and dynamic performance. In particular, for low switching frequency ap-

\footnotetext{
Manuscript received Mar. 25, 2010; revised Jul. 20, 2010

† Corresponding Author: yckim@ @bu.ac.kr

Tel: +82-43-261-2475, Chungbuk Nat'l University

* Chungbuk National University, Korea

${ }^{* *}$ LS Industrial Systems, Korea
}

plications in large power systems, such as traction drives, the digitally implemented cascaded inner/outer structure PI controllers are recommended [10], [11].

In the cascaded structure of the DC output voltage and AC input current controllers of a PWM converter are generally constructed with double feedback loops, which consist of an inner AC current feedback loop and an outer DC voltage feedback loop. Each feedback loop is usually designed with a PI controller. The reference waveform of the AC current is generated by multiplying the error output of the DC voltage controller as the amplitude of the AC current waveform with the unit sinusoidal waveform in phase with the $\mathrm{AC}$ line voltage. Then the AC current controller operates to follow the reference current waveform not only to meet the sinusoidal current waveform and unity power factor but also to regulate the DC voltage. These kinds of current controlled PWM techniques have advantages, such as the control of instantaneous current waveforms and high accuracy, peak current protection, extremely good dynamics, and compensation of effects due to parameter changes and AC source voltage changes, etc. [1], [11]. However the converter includes a switching component, which has nonlinear characteristics, and both the inner loop and the outer loop cannot be handled in a single framework. It could be modeled in a single nonlinear system using a power balance, but the small signal model is valid only around specified operating points [12]. Otherwise, it could be linearized by using input-output feedback, but the design of the included PI controllers was not investigated [13].

This paper presents a new technique for designing direct digital controllers so that they provide good transient re- 
sponses for DC voltage regulation under practical severe load change conditions. The inner current control loop can be designed using the error-space approach [14] independently of the outer voltage loop [15]. Then, the whole inner loop system, including the current controller, can be replaced by an equivalent transfer function. The inner loop system, from the right end of the voltage controller to the capacitor, is identified from the input-output data using the CLOE identification algorithm presented in [16]. Based on identified linear model $\bar{G}_{o}(z)$, a voltage controller satisfying the desired time response specifications is designed, using the $w$-transform [17], [18] and the characteristic ratio assignment (CRA) [19]. The reference transfer function $H^{*}(w)$ that meets the prescribed time response is preliminarily generated in the $w$-domain using the CRA, then this is transformed into the $z$-domain as $H^{*}(z)$ so that a controller is obtained directly in the $z$-domain by solving the Diophantine equation. A similar approach has been presented in [20]. However, the method in [20] can not be referred to the fully direct digital control of a PWM converter because the design of the inner loop controller is carried out by an indirect method in the manner that a continuous-time current controller is first designed and then converted to a digital approximation. In this paper, the fully direct digital design of both the inner and the outer-loop controllers is considered.

For verification of the proposed control algorithm, a prototype of a $4.5 \mathrm{~kW}$ single phase PWM converter is tested considering the application of an urban light railway system. Through the simulation and experimental results, it is shown that the proposed method is useful for the practical design of PWM converter controllers.

This paper is organized as follows. A single-phase AC/DC PWM converter model is introduced in Section II. In Section III, the direct digital controller design method is presented. The design procedures for the inner current control and the outer voltage control for a PWM converter system are presented in Section IV. In Section V, simulation and experimental results will be followed, as a practical example. Finally, some conclusions are given in Section VI.

\section{PWM CONVERTER MOdel}

The configuration of a power circuit for a single-phase PWM converter model is shown in Fig. 1. Figure 2 shows the double loop feedback control system, which consists of an inner AC-current loop and an outer DC-voltage loop.

The current controller $C_{i}$ and the voltage controller $C_{v}$ are used to force the input current to follow the referenced current waveform and to regulate the output voltage even under the condition of a sudden load change.

In general, the current controller is designed so that the power factor at the supply terminal is close to one. That is, it is required that the supply voltage $v_{s}$ and the current $i_{s}$ be in-phase as close as possible. This means that both the current loop and the voltage loop cannot be handled in a single framework because of the nonlinearity due to the $60 \mathrm{~Hz}$ modulator. This is the main reason that a simple controller of the PID type for a PWM converter is difficult to obtain analytically.

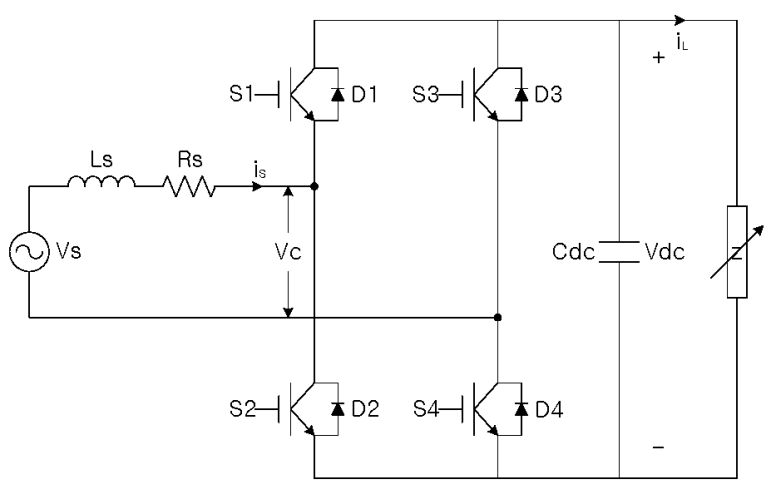

Fig. 1. Typical circuit for a PWM converter.

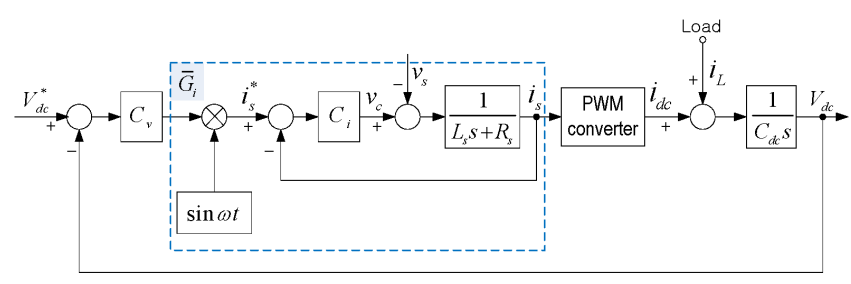

Fig. 2. Block diagram of the PWM converter control system.

In the next section, the design technique of a direct digital controller is introduced, which will meet the given time response specifications in a single loop feedback system. This approach will be used for the digital control problem of the PWM converter.

\section{Direct Digital Control with Time Response REQUIREMENTS}

Consider the discrete-time feedback control system shown in Fig. 3. A linear time invariant (LTI) plant and an R-S-T type controller [16] are described by

$$
\begin{aligned}
& \frac{B\left(z^{-1}\right)}{A\left(z^{-1}\right)}=\frac{b_{1} z^{-1}+b_{2} z^{-2}+\cdots+b_{n_{B}} z^{-n_{B}}}{1+a_{1} z^{-1}+a_{2} z^{-2}+\cdots+a_{n_{A}} z^{-n_{A}}} \\
& S\left(q^{-1}\right) u(k)=-R\left(q^{-1}\right) y(k)+T\left(q^{-1}\right) r(k)
\end{aligned}
$$

where $q$ denotes the shift operator defined by $q y(k):=y(k+$ $1)$, and

$$
\begin{aligned}
& S\left(z^{-1}\right)=1+s_{1} z^{-1}+\cdots+s_{n_{S}} z^{-n_{S}} \\
& R\left(z^{-1}\right)=r_{0}+r_{1} z^{-1}+\cdots+r_{n_{R}} z^{-n_{R}} \\
& T\left(z^{-1}\right)=t_{0}+t_{1} z^{-1}+\cdots+r_{n_{T}} z^{-n_{T}}
\end{aligned}
$$

The closed-loop transfer function is given by

$$
\begin{aligned}
H\left(z^{-1}\right) & =\frac{T\left(z^{-1}\right) B\left(z^{-1}\right)}{A\left(z^{-1}\right) S\left(z^{-1}\right)+B\left(z^{-1}\right) R\left(z^{-1}\right)} \\
& =\frac{T\left(z^{-1}\right) B\left(z^{-1}\right)}{P\left(z^{-1}\right)} .
\end{aligned}
$$

The characteristic polynomial $P\left(z^{-1}\right)$ is

$$
\begin{aligned}
P\left(z^{-1}\right) & =A\left(z^{-1}\right) S\left(z^{-1}\right)+B\left(z^{-1}\right) R\left(z^{-1}\right) \\
& =p_{0}+p_{1} z^{-1}+\cdots+p_{n} z^{n} .
\end{aligned}
$$




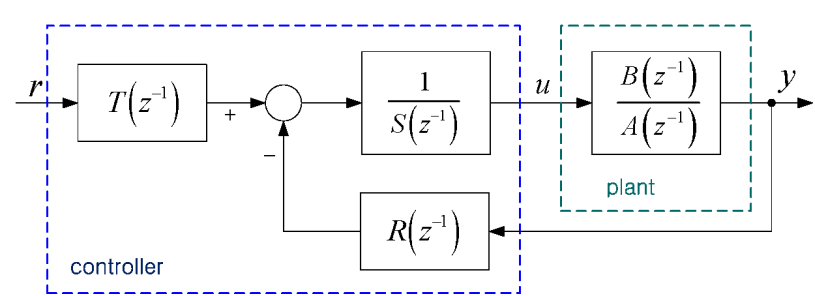

Fig. 3. Feedback system with R-S-T type controller.

Suppose that the design problem here is to find a digital controller $R, S, T$ for the given discrete time response specifications, such as the maximum overshoot and the settling time limitation.

Applying the model matching method to this problem, it can be described by

$$
\begin{aligned}
H\left(z^{-1}\right) & =\frac{T\left(z^{-1}\right) B\left(z^{-1}\right)}{P\left(z^{-1}\right)} \\
& \equiv \frac{T\left(z^{-1}\right) B\left(z^{-1}\right)}{P^{*}\left(z^{-1}\right)}=H^{*}\left(z^{-1}\right) .
\end{aligned}
$$

where $H^{*}\left(z^{-1}\right)$ and $P^{*}\left(z^{-1}\right)$ are the reference model and the reference characteristic polynomial, respectively. To proceed with (6), it is necessary to find a reference model $H^{*}\left(z^{-1}\right)$ that satisfies the time response specifications. In [18], a simple technique for this problem has been proposed. This method consists of three steps. Firstly, the prospective closed-loop transfer function $H\left(z^{-1}\right)$ is transformed into the $w$-domain by the $w$-transform. The transformed function is given by

$$
H(w)=\frac{\bar{B}(w)}{P(w)}=\frac{t_{0} \sum_{i=0}^{n} b_{i}(w+C)^{i}(w-C)^{n-i}}{\sum_{j=0}^{n} p_{j}(w+C)^{j}(w-C)^{n-j}}
$$

where $C=\frac{2}{T_{s}}$.

Here, it is notable that $\bar{B}(w)$ is a fixed known polynomial whose coefficients are functions of only $B\left(z^{-1}\right)$ for a given $T\left(z^{-1}\right)$. The second step is to find a reference polynomial $P^{*}(w)$ so that the reference model $H^{*}(w)=\bar{B}(w) / P^{*}(w)$ meets the design specifications. Such a reference model can be easily found by means of the CRA, as presented in [18]. In the third step, $H^{*}\left(z^{-1}\right)$ is obtained by transforming $H^{*}(w)$ into the $z$-domain inversely. Then the controller, $S\left(z^{-1}\right)$ and $R\left(z^{-1}\right)$ can be determined by solving the following algebraic equation.

$$
\begin{aligned}
P^{*}\left(z^{-1}\right) & =A\left(z^{-1}\right) S\left(z^{-1}\right)+B\left(z^{-1}\right) R\left(z^{-1}\right) \\
& =p_{0}^{*}+p_{1}^{*} z^{-1}+\cdots+p_{n}^{*} z^{n} .
\end{aligned}
$$

This polynomial equation has a unique solution with a minimal degree (when $A\left(z^{-1}\right)$ and $B\left(z^{-1}\right)$ do not have common factors) for $n_{P} \leq n_{A}+n_{B}-1, n_{R}=n_{A}-1$, and $n_{S}=n_{B}-1$.

To achieve zero steady state error to a step reference input, the overall system must be of Type 1 . Then $T\left(z^{-1}\right)$ can be

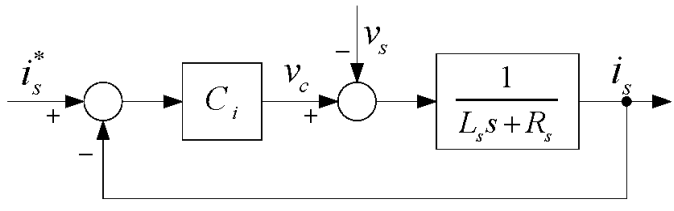

Fig. 4. Block diagram of the inner loop system.

obtained by

$$
T\left(z^{-1}\right)=t_{0}=\frac{P(1)}{B(1)}=R(1) .
$$

\section{PWM Converter Control System Design}

This section describes the design process for the double loop controller. In the first step, the inner current controller is designed, based on the error space approach, independently of the outer control loop. Subsequently, an equivalent linear model of the inner loop system, which includes some nonlinear components, is obtained. After selecting a temporary voltage controller that makes the overall system stable, the CLOE identification method is applied to identify the linearized model from the input-output data. Once the model is identified, a direct digital voltage controller is designed by using the $w$ transform and the CRA method [18].

\section{A. Inner Loop Design}

The error-space feedback scheme is a well known statefeedback method that allows a controller to perfectly track a non-decaying input, and to reject non-decaying disturbances such as sinusoidal inputs. Since this method solves the control problem in the error space, it has robustness because the error approaches zero against the variation of some parameters provided that the system keeps stable.

The state equation of the inner loop system in Fig. 4 can be expressed as

$$
\left\{\begin{array}{l}
\dot{x}(t)=-\frac{R_{s}}{L_{s}} x(t)-\frac{1}{L_{s}}\left[u(t)-v_{s}(t)\right] \\
y(t)=x(t)
\end{array}\right.
$$

where $x(t)=i_{s}(t)$ and $u(t)=v_{c}(t)$.

The discrete-time state equations of (10) with a sample time $T_{s}$ are given by

$$
\begin{cases}x(k+1) & =\Phi x(k)+\Psi \bar{u}(k), \\ y(k) & =x(k)\end{cases}
$$

where

$$
\left\{\begin{array}{l}
\Phi=e^{-\frac{R_{s}}{L_{s}} T_{s}}, \\
\Psi=-\frac{1}{L_{s}} \int_{0}^{T_{s}} e^{-\frac{R_{s}}{L_{s}} t} d t=-\frac{1}{R_{s}}\left[1-e^{-\frac{R_{s}}{L_{s}} T_{s}}\right], \\
\bar{u}(k):=\left[u(k)-v_{s}(k)\right] .
\end{array}\right.
$$

Let the reference input of the current controller be $r(k)=$ $i_{s}^{*}(k)=I_{s} \sin \left(\omega_{0} k T_{s}\right)$. It follows that

$$
\Gamma(q) r(k)=0
$$

where

$$
\Gamma(q)=q^{2}-2 \cos \left(\omega_{0} T_{s}\right) q+1
$$


Letting $\beta=\cos \left(\omega_{0} T_{s}\right)$, then (14) is rewritten as

$$
\Gamma(q)=q^{2}-2 \beta q+1 .
$$

The tracking error is defined as

$$
e(k):=r(k)-y(k)=i_{s}^{*}(k)-i_{s}(k) .
$$

From (13) and (16), the error is formulated in terms of the plant state,

$$
\Gamma(q) e(k)=-\Gamma(q) y(k)=-\Gamma(q) x(k) .
$$

Now, a new state vector and a control input in the error space are defined as

$$
\begin{aligned}
\xi(k) & :=\Gamma(q) x(k), \\
\mu(k) & :=\Gamma(q) u(k) .
\end{aligned}
$$

Then (17), (18), and (19) lead to

$$
\begin{aligned}
\Gamma(q) e(k) & =-\xi(k), \\
\xi(k+1) & =\Phi \xi(k)+\Psi \mu(k) .
\end{aligned}
$$

In the derivation of (21), the relation $\Gamma(q) v_{s}(k)=0$ is used as long as $v_{s}(k)$ keeps the same frequency as $i_{s}^{*}(k)$. Equations (20) and (21) show the overall system in error space. Rewriting them in the state variable form, they can be described as

$$
z(k+1)=F z(k)+G \mu(k)
$$

where $z(k)=\left[\begin{array}{lll}e(k) & e(k+1) & \xi(k)\end{array}\right]^{T}$ and

$$
F=\left[\begin{array}{ccc}
0 & 1 & 0 \\
-1 & 2 \beta & -1 \\
0 & 0 & \Phi
\end{array}\right], \quad G=\left[\begin{array}{l}
0 \\
0 \\
\Psi
\end{array}\right] \text {. }
$$

It is obvious that $\{F, G\}$ is controllable. Therefore, there exists a controller that can assign closed-loop poles to any desired location. Let the state feedback controller be

$$
\mu(k)=-K z(k),
$$

where $K:=\left[\begin{array}{lll}k_{1} & k_{2} & k_{3}\end{array}\right]$.

From (18), (19) and (24), it is shown that

$$
\Gamma(q) u(k)+k_{3} \Gamma(q) x(k)=-k_{1} e(k)-k_{2} e(k+1) .
$$

Then (25) can be rearranged as

$$
\begin{aligned}
& {\left[u(k)+k_{3} x(k)\right] q^{2}-2 \beta\left[u(k)+k_{3} x(k)\right] q} \\
& \quad+\left[u(k)+k_{3} x(k)\right]=-\left(k_{1}+k_{2} q\right) e(k) .
\end{aligned}
$$

Let us define

$$
\eta(k):=u(k)+k_{3} x(k) .
$$

Using (27), (26) yields

$$
\eta(k)=\left[2 \beta \eta(k)-k_{2} e(k)+\eta_{1}(k)\right] q^{-1}
$$

where

$$
\eta_{1}(k)=\left[-\eta(k)-k_{1} e(k)\right] q^{-1} .
$$

Let $\eta(k)=\eta_{2}(k)$, from (27), (28), and (29), the control law is given by

$$
\begin{aligned}
{\left[\begin{array}{l}
\eta_{1}(k+1) \\
\eta_{2}(k+1)
\end{array}\right] } & =\left[\begin{array}{ll}
0 & -1 \\
1 & 2 \beta
\end{array}\right]\left[\begin{array}{l}
\eta_{1}(k) \\
\eta_{2}(k)
\end{array}\right]-\left[\begin{array}{l}
k_{1} \\
k_{2}
\end{array}\right] e(k), \\
u(k) & =\eta_{2}(k)-k_{3} x(k)
\end{aligned}
$$

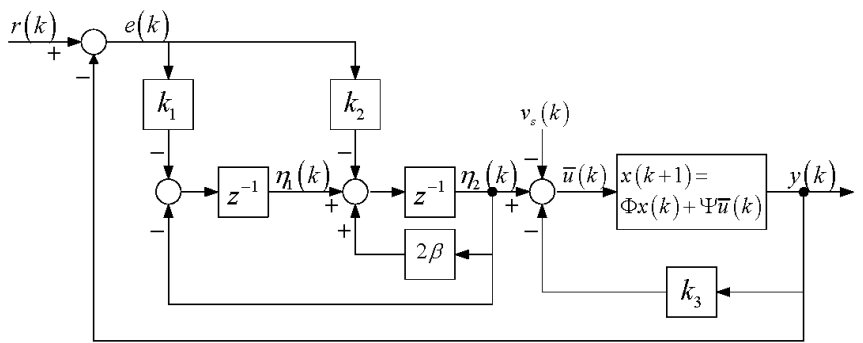

Fig. 5. Current controller for PWM converter.

A block diagram of the current controller law (30) and (31) is shown in Fig. 5. The remaining problem is to determine the controller gain vector $K$ such that it satisfies the given time response requirements.

The closed loop dynamics of the current control loop are determined by combining (30) and (31) with (11) to yield

$$
\left\{\begin{array}{l}
{\left[\begin{array}{c}
x(k+1) \\
\eta_{1}(k+1) \\
\eta_{2}(k+1)
\end{array}\right]=\left[\begin{array}{ccc}
\Phi-\Psi k_{3} & 0 & \Psi \\
k_{1} & 0 & -1 \\
k_{2} & 1 & 2 \beta
\end{array}\right]\left[\begin{array}{c}
x(k) \\
\eta_{1}(k) \\
\eta_{2}(k)
\end{array}\right]} \\
+\left[\begin{array}{c}
0 \\
-k_{1} \\
-k_{2}
\end{array}\right] r(k)+\left[\begin{array}{c}
\Psi \\
0 \\
0
\end{array}\right] v_{s}(k), \\
y(k)=\left[\begin{array}{lll}
1 & 0 & 0
\end{array}\right]\left[\begin{array}{c}
x(k) \\
\eta_{1}(k) \\
\eta_{2}(k)
\end{array}\right]
\end{array}\right.
$$

The closed-loop transfer function from $r(k)$ to $y(k)$ is given by

$$
T_{i}(z)=\frac{I_{s}(z)}{I_{s}^{*}(z)}=\frac{Y(z)}{R(z)}=\frac{-\Psi\left(k_{2} z+k_{1}\right)}{\delta(z)}
$$

where

$$
\begin{array}{r}
\delta(z)=z^{3}+\left(\Psi k_{3}-\Phi-2 \beta\right) z^{2}+\left(-\Psi k_{2}-2 \beta \Psi k_{3}\right. \\
+2 \beta \Phi+1) z+\left(-\Psi k_{1}+\Psi k_{3}-\Phi\right) .
\end{array}
$$

Here, the current controller gain $K$ is easily obtained from $\delta(z) \equiv \delta^{*}(z)$ while the target polynomial $\delta^{*}(z)$ can be generated by the K-polynomial [18] using the CRA approach. The definition of the K-polynomial is referred to the Appendix VI-A.

Remark 1: The design objective of the current control loop is to make the AC supply voltage and the source current in phase. In order to accomplish this purpose, the internal model control (IMC) approach shown in (13) to (24) has been occupied. In this section, $i_{s}^{*}$ and $v_{s}$ in Fig. 2 have been assumed to be sinusoidal waves of $\omega_{0}[\mathrm{rad} / \mathrm{sec}]$. For the cases where these signals include several harmonics due to distortions and sags, there may be some error because the IMC here is used for tracking only one frequency, $\omega_{0}[\mathrm{rad} / \mathrm{sec}]$.

\section{B. Outer Loop Design}

In the previous subsection, it was presented that the current controller can be designed independently of the voltage controller. As shown in Fig. 2, two nonlinear components are included in the outer loop. Since the output voltage must be kept constant, it is possible to characterize this nonlinear system by a linear model, $\bar{G}_{0}\left(z^{-1}\right)$, as shown in Fig. 6 . 


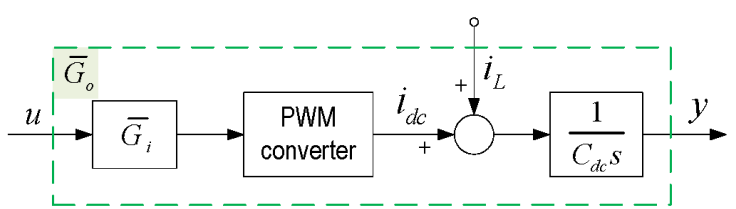

Fig. 6. An equivalent linearized model of the inner loop system.

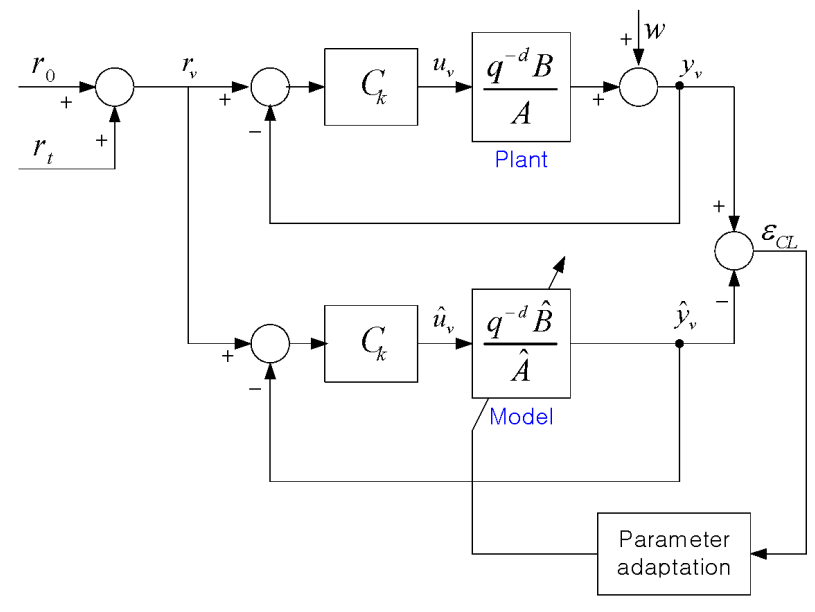

Fig. 7. Closed-loop output error (CLOE) identification method

Because the identification should be carried out while the switching components are in operation, the following closedloop identification scheme is utilized.

1) Closed-loop identification: The principles of the closedloop identification method are illustrated in Fig. 7.

Let the plant to be identified be

$$
\begin{aligned}
\bar{G}_{o}\left(q^{-1}\right) & =q^{-d} \frac{B\left(q^{-1}\right)}{A\left(q^{-1}\right)} \\
& =q^{-d} \frac{b_{1} q^{-1}+\cdots+b_{n_{B}} q^{-n_{B}}}{1+a_{1} q^{-1}+\cdots+a_{n_{A}} q^{-n_{A}}} .
\end{aligned}
$$

In Fig. 7, $C_{k}$ denotes the voltage controller and the hatted letters indicate the identified model.

At this point, the controller $C_{k}$ is not known yet. Thus, in order to carry out the closed loop identification, a simple controller, for example, a proportional controller can be chosen temporarily. It does no matter what $C_{k}$ is chosen provided that it makes the closed loop system stable, so that the input and output data of the closed loop operation can be acquired. In this procedure, a small perturbed test signal should be injected into either the $r$ or $u$ signal. A pseudo-random binary sequences (PRBS), $r_{t}$, is added to the reference DC input. The PRBS is a good test input for identification. Then, the controller output $u_{v}$ and the system output $y_{v}$ are measured to estimate the parameters $\{A, B\}$.

Let us define an unknown parameter vector,

$$
\theta(k)=\left[a_{1}, \cdots, a_{n_{A}}, b_{1}, \cdots, b_{n_{B}}\right]^{T},
$$

and the vector of the measured data,

$$
\begin{aligned}
\phi(k)= & {\left[-y_{v}(k), \cdots,-y_{v}\left(k-n_{A}+1\right),\right.} \\
& \left.u_{v}(k-d), \cdots, u_{v}\left(k-d-n_{B}\right)\right]^{T} .
\end{aligned}
$$

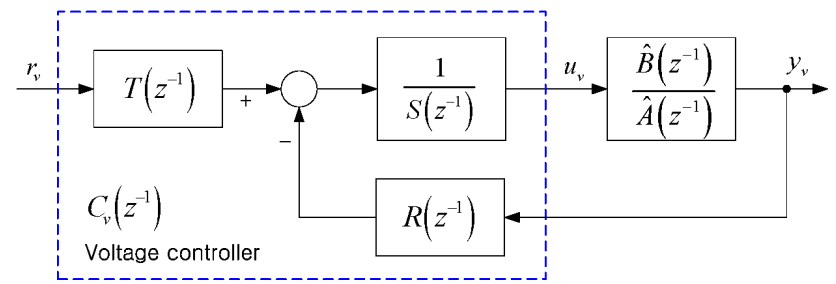

Fig. 8. Closed-loop system of the PWM converter.

Then the a priori predicted output is defined by

$$
\hat{y}_{v}^{0}(k+1)=\hat{\theta}^{T}(k) \phi(k) .
$$

And the estimated controller output is obtained as

$$
\hat{u}_{v}(k)=C_{k}[r(k)-y(k)] .
$$

Furthermore, the a priori closed-loop prediction output error is given by

$$
\hat{\varepsilon}_{C L}^{0}(k+1)=y_{v}(k+1)-\hat{y}_{v}^{0}(k+1),
$$

The parameter adaptation algorithm of the CLOE identification is given in the following recursive form [16].

$$
\begin{aligned}
& \varepsilon_{C L}^{0}(k+1)=y_{v}(k+1)-\hat{\theta}^{T}(k) \phi(k) \\
& \hat{\theta}(k+1)=\hat{\theta}(k)+F(k+1) \Phi(k) \varepsilon_{C L}^{0}(k+1) \\
& F(k+1)=\frac{1}{\lambda_{1}}\left[F(k)-\frac{F(k) \Phi(k) \Phi^{T}(k) F(k)}{\frac{\lambda_{1}}{\lambda_{2}}+\Phi^{T}(k) F(k) \Phi(k)}\right] \\
& \Phi(k)=\phi(k)
\end{aligned}
$$

Herein, the forgetting factors are given by $0<\lambda_{1} \leq 1$, $0<\lambda_{2} \leq 2$.

As a result, the identified equivalent model $\bar{G}_{0}$ is obtained.

2) Voltage Controller Design: Based on the identified model $\hat{G}_{o}\left(z^{-1}\right)=\frac{\hat{B}\left(z^{-1}\right)}{\hat{A}\left(z^{-1}\right)}$, a direct digital controller that satisfies the transient response specifications is designed.

A block diagram of a PWM converter feedback system is shown in Fig. 8.

The main idea of the direct digital design in [18] is to extend the continuous time CRA to the discrete time case. The controller structure of the $R-S-T$ type is considered and it is assumed that the design objective is to directly find a digital controller of fixed order that meets certain time and frequency response requirements.

As explained in Section III, the prospective closed-loop transfer function $H\left(z^{-1}\right)$ is transformed into the $w$-domain by using the $w$-transformation. After regarding $H(w)$ as a function of the $s$-domain $H(s)$, a reference transfer function associated with a fixed numerator $\bar{B}(w)$ is composed by using the CRA technique [21], so that it satisfies the design specifications. It is shown in [18] that the mapping error of the w-transform between the s-and w-domains is less than $3 \%$ for $|w| \leq 0.6 / T_{s}$ and $|s| \leq 0.6 / T_{s}$. In other words, $H(w) \approx H(s)$ is concluded if a sampling time $T_{s}$ is selected so that all the poles and zeros of $H(w)$ lie in the region of $|w| \leq 0.6 / T_{s}$ in left half plane of the w-domain. Once such a reference model $H^{*}(w)=\bar{B}(w) / P^{*}(w)$ is obtained, then 
TABLE I

THE PARAMETERS OF THE MODEL

\begin{tabular}{c|c}
\hline System parameters & values \\
\hline Input voltage $\left(V_{s}\right)$ & $150 \mathrm{~V}$ (peak), $60 \mathrm{~Hz}$ \\
Output voltage $\left(V_{D C}\right)$ & $200 \sim 300 \mathrm{~V}$ \\
Switching frequency & $540 \mathrm{~Hz}$ \\
Sampling frequency & $1080 \mathrm{~Hz}$ \\
Resistor & $0.08 \Omega$ \\
Inductor $\left(L_{s}\right)$ & $1 \mathrm{mH}$ \\
Capacitor $\left(C_{D C}\right)$ & $6000 \mu \mathrm{F}$ \\
Load $\left(R_{L}\right)$ & $12 / 24 \Omega$ \\
\hline
\end{tabular}

$P^{*}(w)$ is transformed into the $z$-domain using the inverse $w$ transformation, which results in $P^{*}\left(z^{-1}\right)$.

$$
P^{*}\left(z^{-1}\right)=1+p_{1}^{*} z^{-1}+\cdots+p_{n}^{*} z^{-n} .
$$

Solving the following Diophantine equation with $P^{*}\left(z^{-1}\right)$ from above, the digital controller of (3) is determined.

$$
P^{*}\left(z^{-1}\right)=\hat{A}\left(z^{-1}\right) S\left(z^{-1}\right)+\hat{B}\left(z^{-1}\right) R\left(z^{-1}\right) .
$$

\section{Simulation And ExPERIMENTAl Results}

Both simulation and experimental demonstrations have been carried out for a single-phase PWM converter model, the parameters of which are given in Table I. This converter has been designed as a laboratory model of a AC/DC converter for using as a high power urban light railway application with a low switching frequency which has a 860 1040 V AC input and a $1600 \mathrm{~V}$ DC output voltage.

The design specifications are as follows:

(i) the maximum settling time of the current loop system is $10 \mathrm{msec}$,

(ii) the overshoot limitation of the DC output is less than $5 \%$,

(iii) the minimum rising time and the maximum settling time of the overall system are $20 \mathrm{msec}$ and $200 \mathrm{msec}$, respectively,

(iv) the stability margins are $G M \geq 10 d B$ and $P M \geq$ 45 deg.

As a simulation tool, the PSiM utility was used. Figure 9 shows the experimental setup of a converter system.

\section{A. Design of the inner loop controller}

As described in Section IV-A, in order to design the current controller gain $K$ using the CRA, it is necessary to make a reference characteristic polynomial $\delta^{*}(z)$ satisfying a settling time of $10 \mathrm{msec}$. According to the CRA method in [18], when $\alpha_{1}=3$ and $\tau=3.2 \times 10^{-3}$ are selected, such a reference polynomial in the w-domain is obtained as follows:

$$
\delta^{*}(w)=w^{3}+2.8125 \times 10^{3} w^{2}+2.637 \times 10^{6} w+8.24 \times 10^{8} .
$$

The detailed derivation of (43) is referred to Appendix VI-B. The inverse w-transformed polynomial $\delta^{*}(z)$ is given by

$$
\delta^{*}(z)=z^{3}-1.184 z^{2}+0.4673 z-0.00615 .
$$

Solving the identity $\delta(z) \equiv \delta^{*}(z)$ from (34) and (44), the current controller gain $K$ is determined by

$$
K=\left[\begin{array}{lll}
k_{1} & k_{2} & k_{3}
\end{array}\right]=\left[\begin{array}{lll}
-0.8480 & 0.8674 & -1.8196
\end{array}\right] .
$$

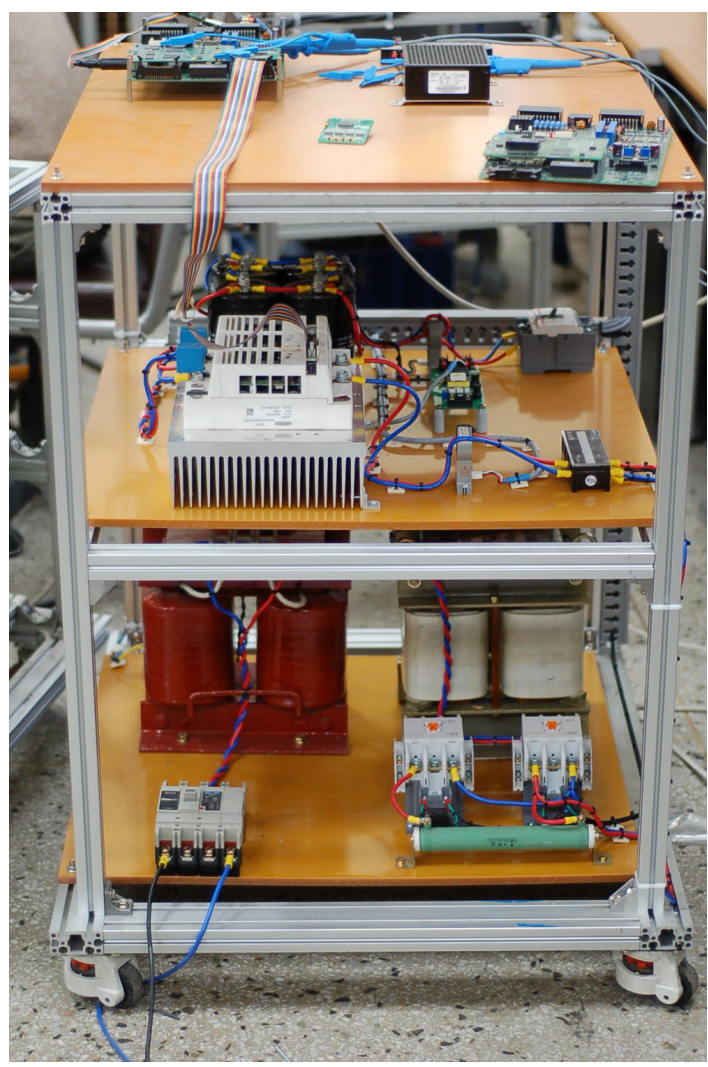

Fig. 9. Experimental setup of the PWM converter.

As a result of (44) and (45), the inner loop control system has three poles at $0.3947,0.3947 \pm j 3.59 \times 10^{-6}$ and a zero at 0.9776 , respectively. It is easy to see from the Bode plot that the bandwidth of the inner loop system is about $f_{B W}=309 \mathrm{~Hz}$. Generally, the sampling frequency can be chosen by the rule [16], $f_{s}=(6 \sim 25) \times f_{B W}$, where $f_{s}$ and $f_{B W}$ are the sample frequency and the closed loop system bandwidth, respectively. Therefore, the sample frequency for the inner loop system needs to be greater than $1,854 \mathrm{~Hz}$. However, the sample frequency given in this example is $1,080 \mathrm{~Hz}$ which is much lower than the recommended value. This is a case where the direct digital control prefers to the digitization approach [16]. This inner loop controller was simulated using the PSiM toolbox. As shown in Fig. 10, the controller makes the current $i_{s}$ and the supply voltage $v_{s}$ be in phase. The total harmonic distortion (THD) of the current $i_{s}$ obtained at the steady state by this controller was less than $0.45 \%$. Fig. 11 shows that the current controller accomplishes perfect tracking within $10 \mathrm{msec}$.

\section{B. Design of the outer loop controller}

Before going to design a voltage controller, an equivalent model of the outer loop has to be identified. To do this, a proportional voltage controller was temporarily selected. Using the CLOE identification method described in Section IV-B2, the linear equivalent model is obtained by

$$
\hat{G}\left(z^{-1}\right)=\frac{0.04227 z^{-1}}{1-z^{-1}} .
$$




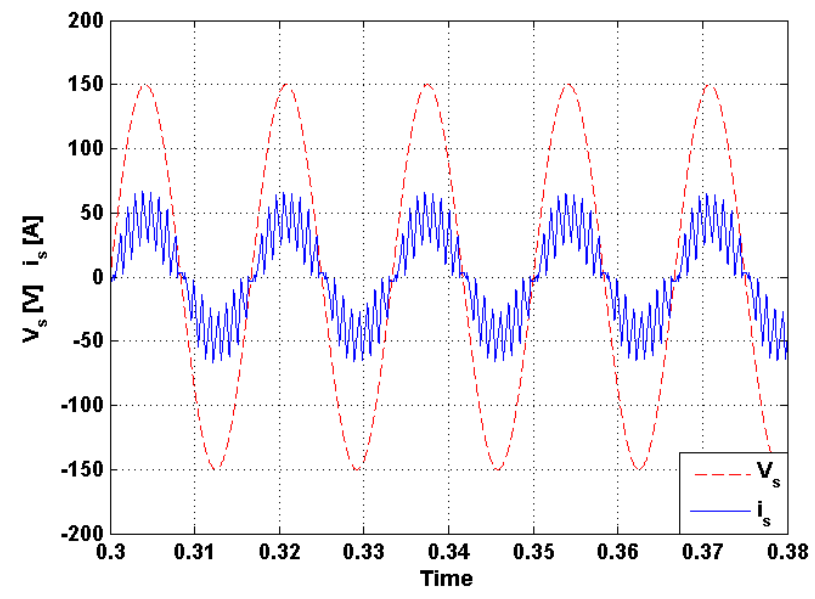

Fig. 10. The simulation results of the $v_{s}$ and $i_{s}$.

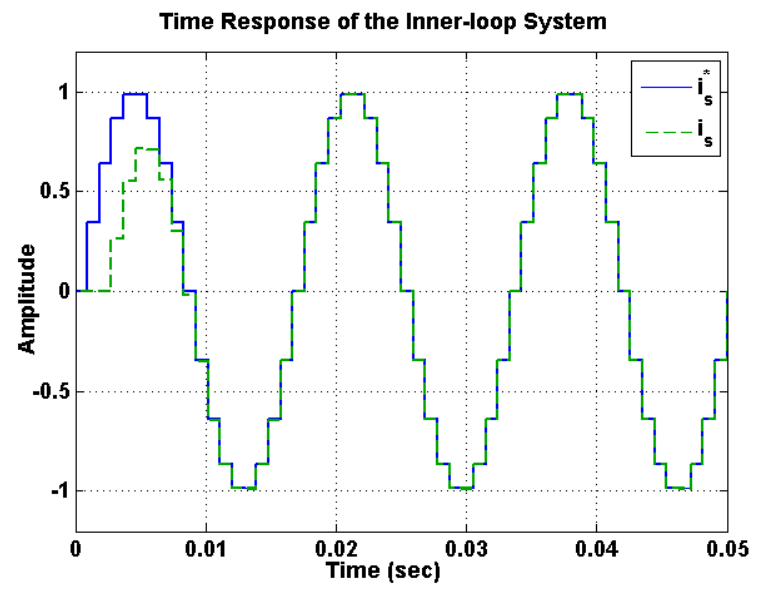

Fig. 11. Time responses of the $i_{s}^{*}$ and $i_{s}$.

A PRBS test signal of $\pm 10 \mathrm{~V}$ for identification was added to the reference input $r$ (where $r=200 \mathrm{~V}$ DC), as shown in Fig. 12-(a). Fig. 12-(b) shows the actual output data and the estimated output, while the bottom curve shows the residual which is the difference between the output of the real system and that of the estimated model.

Fig. 13 shows the profiles about how the identified parameters in the CLOE algorithm converge.

Since the equivalent model (46) is of the first order, a $R S T$ type PI controller is considered for an outer loop controller. Similar to the design of the inner loop controller, the reference characteristic polynomial $P^{*}(z)$ below is obtained, so that it meets a settling time of $200 \mathrm{msec}$ and no overshoot.

$$
P^{*}(z)=z^{2}-1.9273 z+0.9286 .
$$

By solving (42) with (47), the voltage controller is

$$
\begin{aligned}
& R\left(z^{-1}\right)=1.7205-1.6893 z^{-1}, \\
& S\left(z^{-1}\right)=1-z^{-1}, \\
& T\left(z^{-1}\right)=0.0313 .
\end{aligned}
$$

The step and frequency responses of the closed-loop system are shown in Figs. 14 and 15, respectively.
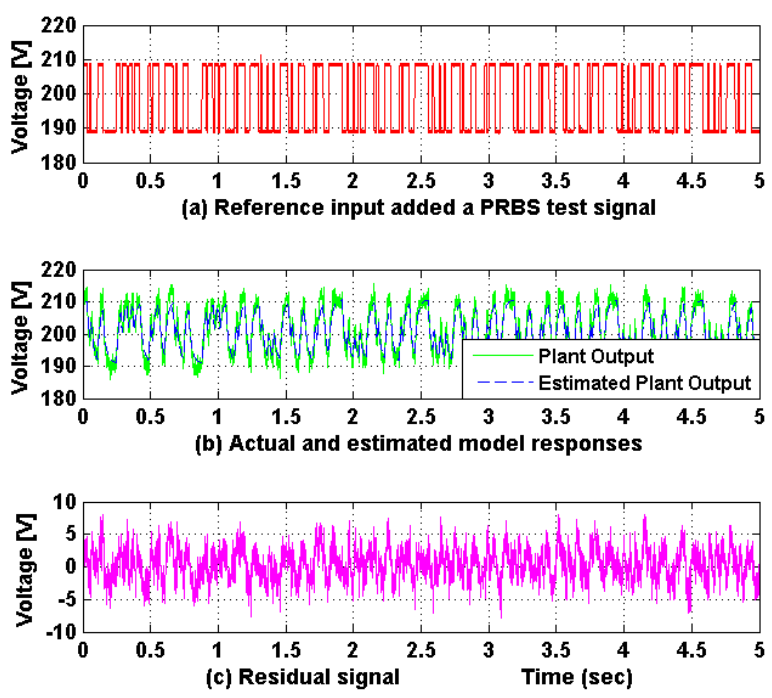

Fig. 12. Time response of the estimated output.
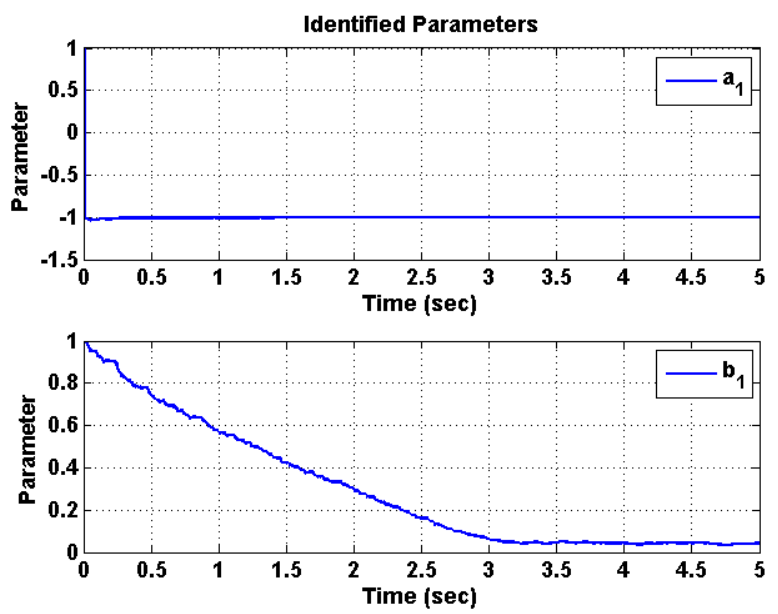

Fig. 13. Convergence of the identified parameters.

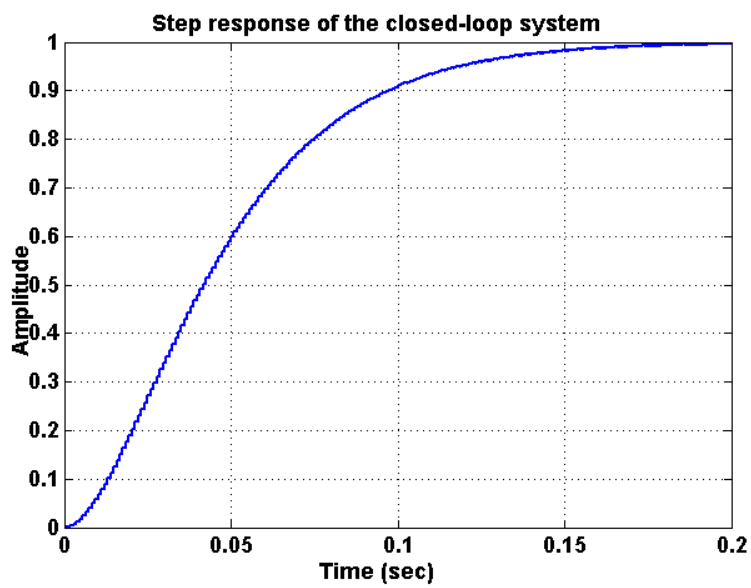

Fig. 14. Step response of the closed-loop system.

The overall system results in no overshoot, a rising time of $84 \mathrm{msec}$ and a settling time of $145 \mathrm{msec}$. Also, both the 


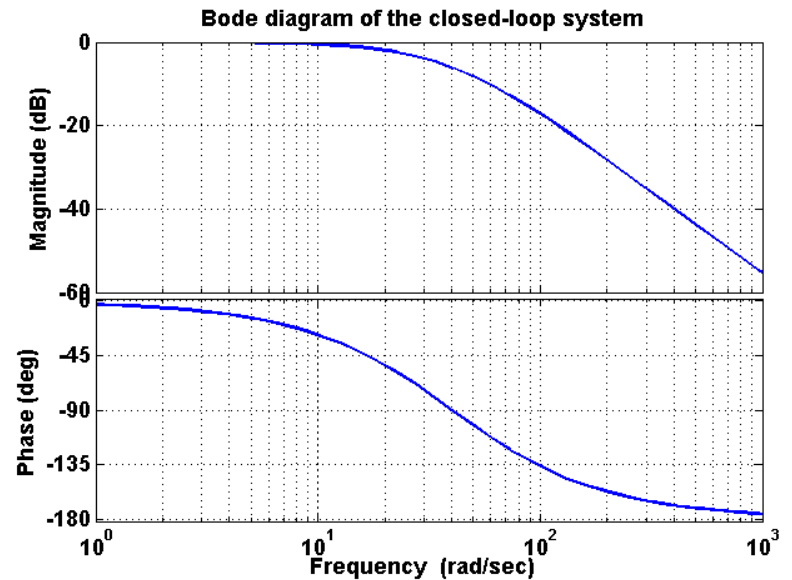

Fig. 15. Bode diagram of the closed-loop system.

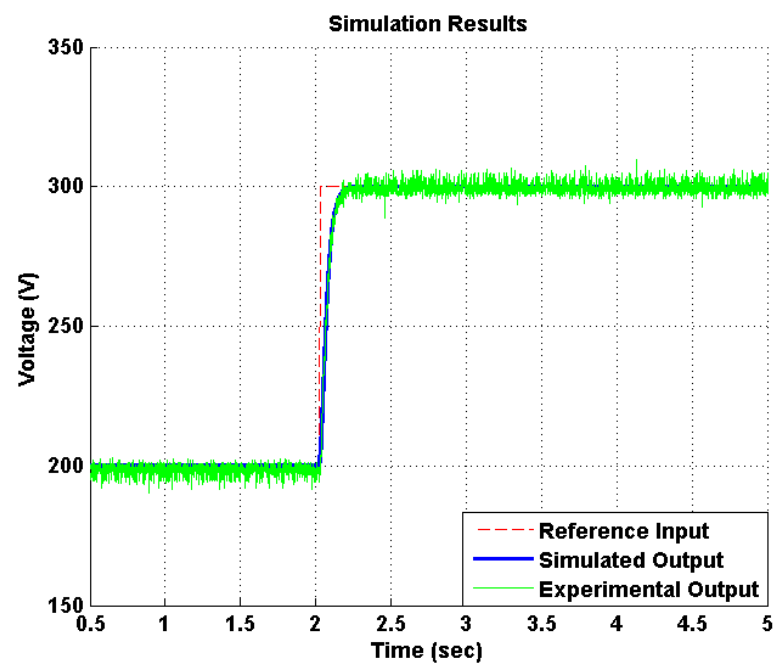

Fig. 16. Simulated and experimental input and output voltages.

gain and the phase margins for stability are $69.3 d B$ and $\infty$, respectively. From these results, it has been verified that the time and stability performances are satisfactory.

Figure 16 shows both the simulation and experimental results for the case where the reference voltage is changed from $200 \mathrm{VDC}$ to $300 \mathrm{VDC}$ at $t=2 \mathrm{sec}$. Both responses coincide with each other and have almost no overshoot.

The time domain performance, subject to abrupt load changes, was experimentally examined. When the load is changed from no load to a half load ar $t=1.25 \mathrm{sec}$, from a half to full load at $t=2.3 \mathrm{sec}$, and from full load to no load at $t=3.3 \mathrm{sec}$, the output voltage consistently remains the same as the reference voltage, as shown in Fig. 17. In this case, the maximum overshoot is less than $4.6 \%$. Figure 18 shows the experimental results of $v_{s}$ and $i_{s}$ which correspond to those of Fig. 10. It is seen that these experimental curves are very similar to the simulated ones.

\section{CONCLUSION}

In this paper, a direct digital controller design method for a single-phase AC/DC PWM converter system, using
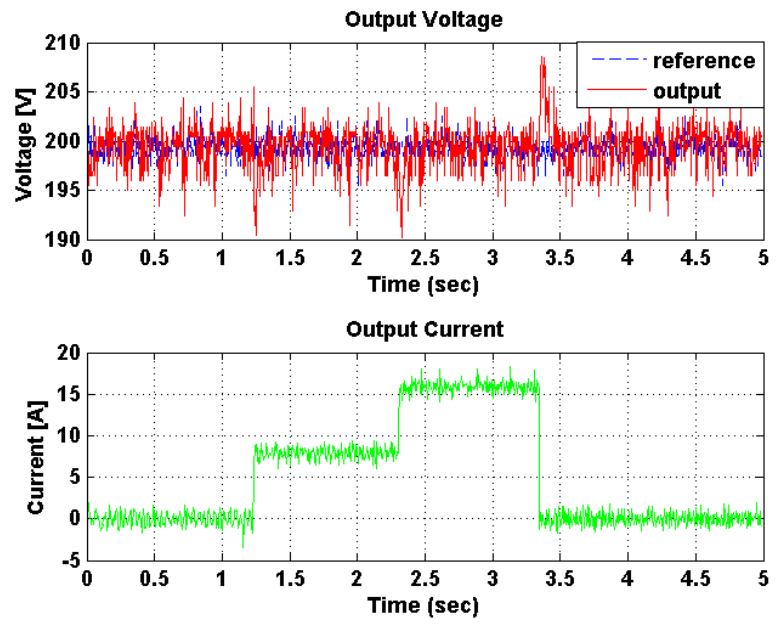

Fig. 17. The output voltages with respect to the load changes.

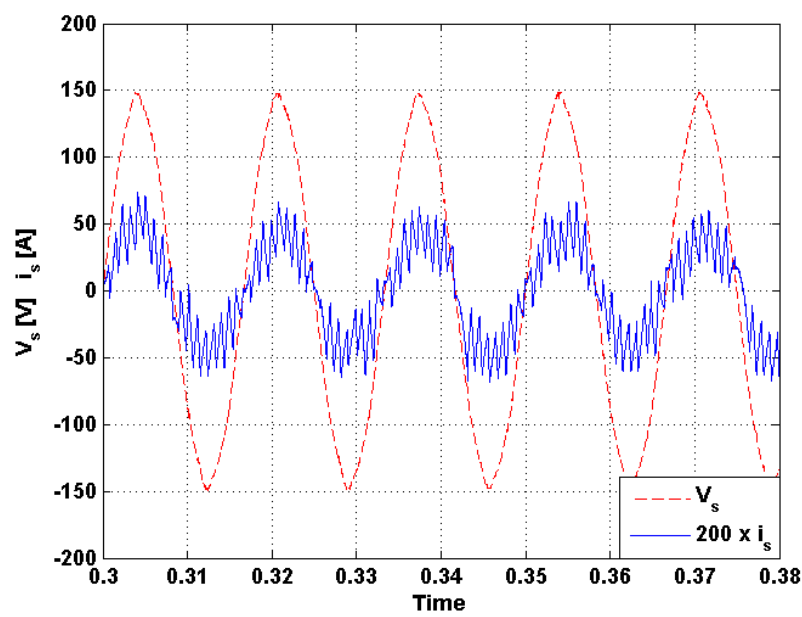

Fig. 18. The experimental results of the $v_{s}$ and $i_{s}$ corresponding to Fig. 10.

closed-loop identification, has been proposed. An error space approach, with the CRA method, is applied for the current control. To identify the linearized model for the outer loop system, which includes an inner loop controller and a switching circuit for the nonlinear component, the CLOE identification method is adapted. Then, the $w$-transform and the CRA method are used to design a direct digital controller for the identified model. The converter controller is designed to meet the specified time response performances, even though the operational conditions are changed. The PSiM simulations and experiments have been carried out to verify the performance of the designed converter system. From the results, it is shown that the new proposed method achieves good performance.

\section{APPENDIX}

\section{A. Definition of the K-polynomial}

Consider a real polynomial with positive coefficients,

$$
\delta(s)=\delta_{n} s^{n}+\cdots+\delta_{1} s+\delta_{0}, \text { for } \delta_{k}>0 .
$$


The characteristic ratios and the generalized time constant [21] are defined as

$$
\begin{aligned}
\alpha_{1}:=\frac{\delta_{1}^{2}}{\delta_{0} \delta_{2}}, \quad \alpha_{2}:= & \frac{\delta_{2}^{2}}{\delta_{1} \delta_{3}}, \cdots, \quad \alpha_{n-1}:=\frac{\delta_{n-1}^{2}}{\delta_{n-2} \delta_{n}}, \\
\tau & :=\frac{\delta_{1}}{\delta_{0}} .
\end{aligned}
$$

According to (50) and (51), the coefficient of $\delta(s)$ can be represented in terms of $\alpha_{i}$ s, and $\tau$ as follows.

$$
\delta_{1}=\delta_{0} \tau, \quad \delta_{2}=\frac{\delta_{0} \tau^{2}}{\alpha_{1}}, \cdots, \delta_{n}=\frac{\delta_{0} \tau^{n}}{\alpha_{n-1} \cdots \alpha_{1}^{n-1}} .
$$

In [21], it has been shown that $\tau$ is related to the speed of the time response of the all pole system whose denominator is $\delta(s)$, and the $\alpha_{i}$ s are closely related to damping and stability.

The K-polynomial is defined as a polynomial for which the characteristic ratios obey the following formula:

$$
\left\{\begin{array}{l}
\alpha_{1} \geq 2 \\
\alpha_{k}=\frac{\sin \left(\frac{k \pi}{n}\right)+\sin \left(\frac{\pi}{n}\right)}{2 \sin \left(\frac{k \pi}{n}\right)} \alpha_{1}, \text { for } k=2, \cdots n-1 .
\end{array}\right.
$$

It is important to note that the $K$-polynomial is generated by only two parameters $\alpha_{1}$ and $\tau$ for any $\delta_{0}$. For example, let us make a $K$-polynomial, $\delta_{k}(s)$, of degree $n=6$ with $\delta_{0}=1$ and $\tau=1$. If we choose $\alpha_{1}=2.5$ arbitrarily, then (53) results in

$$
\left[\begin{array}{llll}
\alpha_{1} & \alpha_{2} & \cdots & \alpha_{5}
\end{array}\right]=\left[\begin{array}{lllll}
2.5 & 1.9717 & 1.875 & 1.9717 & 2.5
\end{array}\right] .
$$

Using (52), the following K-polynomial is obtained.

$$
\begin{aligned}
& \delta_{k}(s)=1.058 \times 10^{-5} s^{6}+4.818 \times 10^{-4} s^{5} \\
& \quad+8.780 \times 10^{-3} s^{4}+8.115 \times 10^{-2} s^{3}+0.4 s^{2}+s+1 .
\end{aligned}
$$

\section{B. Synthesis of the reference polynomial $\delta^{*}(w)$ : Derivation of} (43)

As mentioned in Section IV-A, a current controller gain $K$ that satisfies the maximum settling time and exhibits good damping can be algebraically determined only if such a reference polynomial $\delta^{*}(z)$ is given. Thus, this problem boils down to the problem of finding such a $\delta^{*}(w)$. In Section 3.1 of [18] and Section 6 of [19], the methods for synthesizing a transfer function under the conditions of the transient response requirements have been presented. Here, it will be shown how (43) is obtained by using the CRA method. In this approach, we first choose a characteristic ratio, $\alpha_{1}$, for the $K$-polynomial of degree 3 such that the transfer function $H_{k}(s)=\delta_{0} / \delta_{k}(s)$ has a satisfactory damping. It was shown in [19] that any value of $H_{k}(s)$ s has a non-overshooting step response only if $\alpha_{1}>2.836$ in (53) holds when $n=3$. Thus, we are supposed to choose $\alpha_{1}=3.0$. Then the other characteristic ratio $\alpha_{2}$ becomes 3.0 from (53). Assuming that $\tau_{f}=0.01$ and $\delta_{0}=1$ for the first trial, the corresponding K-polynomial is obtained from (52) as follows;

$$
\delta_{k}\left(s, \tau_{f}=0.01\right)=s^{3}+900 s^{2}+2.7 \times 10^{5} s+2.7 \times 10^{7} .
$$

The step response of the transfer function, $H_{k}\left(s, \tau_{f}=\right.$ $0.01)=2.7 \times 10^{7} / \delta_{k}\left(s, \tau_{f}\right)$, is shown in Fig. 19. It has no overshoot but the settling time of $1 \%$ is $28[\mathrm{msec}]$.

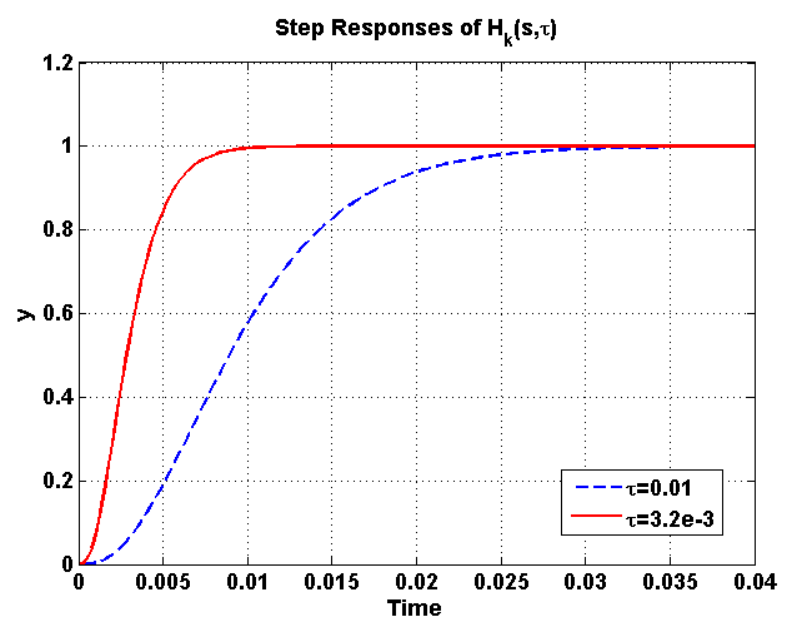

Fig. 19. Step responses of $H_{k}(s) \mathrm{s}$ with two $\tau \mathrm{s}$.

It has been demonstrated in [18] and [19] that the speed of the step response of a linear all pole system can be controlled by adjusting the generalized time constant $(\tau)$ only if all of its characteristic ratios are unchanged. The smaller the value of $\tau$, the faster the settling time becomes while its maximum overshoot holds the same. Suppose that $H_{k}\left(s, \tau_{f}\right)$ gives rise to a settling time of $t_{s f}$. According to the results in [18] and [21], the value of $\tau_{d}$ for $H_{k}\left(s, \tau_{d}\right)$ to have the desired settling time, $t_{s d}$, can be determined by

$$
\tau_{d}=\frac{t_{s d}}{t_{f}} \tau_{f}
$$

Since the desired settling time should be less than $10[\mathrm{msec}]$, we choose $t_{s d}=9[\mathrm{msec}]$. The above equation results in the following $\tau_{d}$.

$$
\tau_{d}=\frac{9 \times 10^{-3}}{28 \times 10^{-3}} \quad(0.01)=3.2 \times 10^{-3} .
$$

Using (52) associated with $\alpha_{1}, \alpha_{2}$, and $\tau_{d}$, the following $K$ polynomial is obtained.

$$
\delta^{*}\left(s, \tau_{d}\right)=s^{3}+2.8125 \times 10^{3} s^{2}+2.637 \times 10^{6} s+8.24 \times 10^{8} .
$$

The step response of the transfer function, $H_{k}\left(s, \tau_{d}\right)=$ $8.24 \times 10^{8} / \delta^{*}\left(s, \tau_{d}\right)$, is also shown in Fig. 19. It is evident that $H_{k}\left(s, \tau_{d}\right)$ is non-overshooting and that it has a settling time of $9[\mathrm{msec}]$. If the complex variable $s$ in (58) is replaced by $w$, (58) is identical to (43). Fig. 11 shows the reference input $i_{s}^{*}$ and the output $i_{s}$ of the closed loop transfer function in (33) of which the denominator has been transformed by $\delta^{*}(w)$. So, we confirm that the resulting current controller exhibits non-overshooting and a settling time of about $8.3[\mathrm{msec}]$.

\section{ACKNOWLEDGMENT}

This work was supported in part by an Ministry of Knowledge Economy, Korea, under the Information Technology Research Center (ITRC) support program supervised by the National IT Industry Promotion Agency (NIPA) (NIPA-2009(C1090-0904-0007)) and in part by a research grant from Chungbuk National University in 2008. 


\section{REFERENCES}

[1] Marian P. Kazmierkowski and Luigi Malesani, "Current control techniques for three-phase voltage-source PWM converters: A survey," IEEE Transactions on Industrial Electronics, Vol. 45, No. 5, pp. 691-703, Oct. 1998.

[2] D.M. Brod and D.W. Novotny, "Current control of VSI-PWM inverters," IEEE Transactions on Industry Applications, Vol. IA-21, No. 3, pp. 562570, May 1985.

[3] W. Leonhard, Control of Electrical Drives, 2nd ed., Springer-Verlag, 1996.

[4] D.C. Lee, S.K. Sul, and M.H. Park, "High performance current regulator for a field-oriented controlled induction motor drive," Conf. Rec. of IEEE IAS Annual Meeting, Vol. 1, pp. 538-544, 1992.

[5] G. Pfaff, A. Weschta, and A. Wick, "Design and experimental results of a brushless ac servo drive," IEEE Transactions on Industry Applications, Vol. IA-22, No. 4, pp. 814-821, Jul. 1984.

[6] K.P. Gokhale, A. Kawamura, and R.G. Hoft, "Deadbeat microprocessor control of PWM inverter for sinusoidal output waveform synthesis," IEEE Transactions on Industry Applications, Vol. IA-23, No. 5, pp. 901909, Sep. 1987.

[7] G. Venkataramanan, D.M. Divan, and T.M. Jahans, "Discrete pulse modulation strategies for high frequency inverter systems," Conf. Rec. of IEEE IPESC'89, pp. 1013-1020, 1989.

[8] B. Burton, R.G. Harley, G. Diana, and J.R. Rodgerson, "Implementation of a neural network to adaptively identify and control VSI fed induction motor stator currents," Conf. Rec. of IEEE IAS Annual Meeting, pp. 1734-1740, 1994.

[9] M.A. Dzieniakowski and P.Z. Grabowski, "Fuzzy logic controller with state recognition for three phase PWM-VSI," Conf. Rec. of IEEE ISIE'96, pp. 438-443, 1996.

[10] X. Huang, X. Wang, T. Nergaard, J.S. Lai, X. Xu, and L. Zhu, "Parasitic ringing and design issues of digitally controlled high power interleaved boost converters," IEEE Transactions on Power Electronics, Vol. 19, No. 5, pp. 1341-1352, Sep. 2004.

[11] D.G. Holmes, B.P. McGrath, D. Segaran, and W.Y. Kong, "Dynamic control of a $20 \mathrm{~kW}$ interleaved boost converter for traction applications," Conf. Rec. of IEEE IAS Annual Meeting, pp. 1-8, 2008.

[12] S. Fukuda, Y. Iwaji, and T. Aoyama, "Modeling and control of sinusoidal PWM rectifiers," Proc. of EPE'1993, Vol. 1, pp. 115-120, 1993.

[13] D.C. Lee, G.M, Lee, and K.D. Lee, "DC-bus voltage control of threephase AC/DC PWM converters using feedback linearization," IEEE Transactions on Industry Applications, Vol. 36, No. 3, pp. 826-833, May/Jun. 2000.

[14] Y.T. Woo and Y.C. Kim, "Digital control of a single-phase UPS inverter for robust AC-voltage tracking," International Journal of Control, Automation, and Systems, Vol. 3, No. 4, pp. 620-2630, Dec. 2005.

[15] J. Choi, S.C. Kim, H.C. Kim, and Y.C. Kim, "CRA based robust digital current controller for AC/DC PWM converter," Conf. Rec. of PCCNagoya, pp. 51-56, 2007.

[16] I.D. Landau and Z. Gianluca, Digital Control Systems, Springer, 2006.

[17] C.H. Houpis and B.L. Gary, Digital Control Systems, McGraw-Hill, 1992.

[18] Y.C. Kim and L.S. Lim, "A parametric design method of direct digital control with transient response requirements," Proc. of the ICCAS-SICE 2009, Fukuoka, Japan, Aug. 2009.

[19] L.H. Keel, Y.C. Kim, and S.P. Bhattacharyya, "Ch.6 Transient response control," Lecture note on the 17th IFAC World Congress Tutorial Workshop on Advances in Three Term Control, Seoul, Korea, Jul. 2008.
[20] Y.C. Kim, L.S. Lim, Lihua Jin, J.M. Lee, and J.H. Choi, "Direct digital control of PWM converter using closed-loop identification," Proc. of the IEEE International Symposium on Industrial Electronics, Seoul, Korea, Jul. 2009.

[21] Y.C. Kim, L.H. Keel, and S.P. Bhattacharyya, "Transient response control via characteristic ratio assignment," IEEE Transactions on Automatic Control, Vol. 48, No. 12, pp. 2238-2244, Dec. 2003.

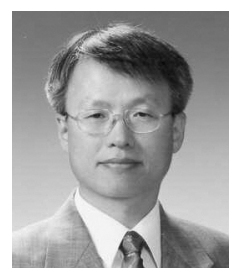

Young Chol Kim received his B.S. in Electrical Engineering from Korea University, Korea in 1981, and his M.S. and the Ph.D. in Electrical Engineering from Seoul National University, Korea in 1983 and 1987 respectively. Since March 1988, he has been with the Colleage of Electrical \& Computer Engineering, Chungbuk National University, Korea, where he is currently a professor. He was a Post-doctoral Fellow at Texas A\&M University in 1992 and a visiting research fellow

at COE-ISM, Tennessee State Univ./Vanderbilt Univ. in 2001. He has been the chairman of the KIEE Control and Instrument Society since 2009. His research interests include parametric robust control, low-order controller design, and transient response control via characteristic ratio assignment.

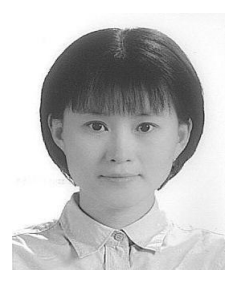

Lihua Jin was born in Jilin, China in 1978. She received her B.S. in Electronic Engineering from Yanbian University, China in June 1999, and her M.S. in Electronics Engineering from Chungbuk National University, Korea in August 2007. She is currently working towards her $\mathrm{Ph} . \mathrm{D}$. in Electronics Engineering. Her research interests include low-order controller design with time response specifications, model-free/non-parametric model design, and system identification.

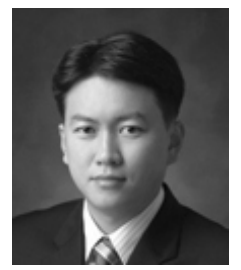

Jinmok Lee received his B.S., M.S., and Ph.D in Electrical Engineering from Chungbuk National University in Cheongju, Korea in 2002, 2004, and 2009, respectively. Currently, he is working at the Automation R\&D Center of LS Industrial Systems as Research Engineer. His research interests include inverter systems, digital control and renewable energy systems. He is a member of KIPE and KIEE.

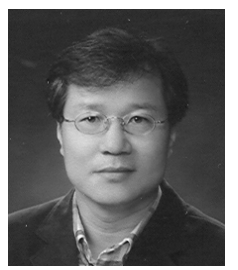

Jaeho Choi received his B.S., M.S., and Ph.D in Electrical Engineering from Seoul National University, Seoul, Korea in 1979, 1981, and 1989, respectively. From 1981 to 1983, he was with Jungkyoung Technical College, Daejeon, Korea, as a Full-time Lecturer. Since 1983, he has been with the School of Electrical and Computer Engineering, Chungbuk National University in Cheongju, Korea, where he is currently a professor. In 1993, 1998 and 2003, he was a visiting Professor at the University of Toronto, Canada, for one each year and he was a Danfoss Visiting Professor at Alborg University in Denmark in 2000. His research interests include power electronics, power quality problems and solutions, energy storage systems, as well as renewable energy and microgrid systems. $\mathrm{He}$ is an active member of KIPE, KIEE, and IEEE, and currently the Editorin-Chief of JPE. 